\section{Ergebnisse einer Studie zur ambulanten Akupunktur- behandlung von alkohol- und medikamentenabhängigen Klienten in einer Beratungsstelle}

Zusammenfassung: Behandlungsbegleitend wurden 228 alkoholabhängige Klienten, die an einer ambulanten Suchtakupunkturbehandlung (Ohrakupunktur mit drei bis fünf Ohrpunkten je Ohr) teilnahmen, hinsichtlich der Merkmale Alkoholkonsum, psychisches und somatisches Befinden befragt. Längerfristige Effekte der Behandlung, die Inanspruchnahme weiterführender Behandlungsmaßnahmen, subjektive Einstellungen und Bewertungen der Akupunktur (Kausalattributionen etc.) wurden katamnestisch (Rücklaufquote: $35,4 \%$ ) untersucht. Die Ergebnisse der Studie, die im Auftrag der Martha-Stiftung zur Vorbereitung einer größeren Evaluationsstudie durchgeführt wurde, zeigen Verbesserungen aller erhobenen Ergebnisvariablen. Der am Ende der Behandlung erreichte Status des psychischen und somatischen Befindens konnte im Katamnesezeitraum gehalten werden, während sich das Konsumverhalten im Katamnesezeitraum verschlechterte. Das Konsumverhalten war dennoch zum Zeitpunkt der Nachbefragung signifikant besser als zu Beginn der Behandlung. Der Therapieerfolg steht außer mit der Anzahl der Behandlungssitzungen mit keiner der untersuchten Rahmenbedingungen der Therapie in einem statistisch signifikantem Zusammenhang.

Schlüsselwörter: Alkoholabhängigkeit - Ambulante Therapie - Akupunktur

Results of a Study on Outpatient Acupuncture Treatment of Alcohol and Drug Dependent Clients at a Counseling Center: In accompaniment to their treatment, 228 alcohol dependent clients participating in an outpatient acupuncture therapy for substance addiction (aural acupuncture with three to five points on each ear) were surveyed concerning characteristics of their alcohol consumption and their psychological and somatic conditions. Long-term effects of the treatment, participation in further treatment measures, subjective attitudes, and evaluations of the acupuncture treatment (causal attributions etc.) were catamnestically examined (returned questionnaires: $35.4 \%$ ). The results of the study, which was performed within the framework of a commission by the Martha-Stiftung for the preparation of a larger evaluation study, show improvements in all of the result variables which were analyzed. The status of the psychological and somatic well-being achieved at the end of the treatment was able to be maintained during the catamnesis

Suchttherapie 2001; 2: 35-44

(c) Georg Thieme Verlag Stuttgart · New York

ISSN 1439-9903
Henry Buhk ${ }^{1}$, Wiebke Busche ${ }^{1}$, Johanne Feldkamp², Uwe Koch ${ }^{1}$

${ }^{1}$ Universitätsklinikum Hamburg-Eppendorf, Medizinische Klinik und Poliklinik, Abteilung für Medizinische Psychologie

2 Ambulante Beratungs- und Behandlungsstelle „Die Hummel“ im Sozialtherapeutischen Zentrum für Suchtkranke, Trägerin Martha-Stiftung

period, while the consumption behavior worsened during the same period. The consumption behavior was however significantly better at the time-point of the follow-up interview than at the beginning of the treatment. The success of the therapy does not have a statistically significant relationship to the examined therapy conditions with the exception of the number of treatment sessions in which the patients participated.

Key words: Alcohol Dependence - Outpatient Therapy Acupunture

\section{Einleitung}

Eines der zentralen Probleme der Behandlung der Alkoholabhängigkeit besteht in der geringen Zahl von Abhängigen, die durch spezifische fachgerechte Behandlungsangebote erreicht werden. So nimmt nach Berechnungen von Feuerlein, Küfner \& Soyka [1] zwar die Mehrheit der Alkoholabhängigen bei Ärzten professionelle Hilfe in Anspruch, aber nur etwa 8,3\% werden pro Jahr alkoholspezifisch (ambulant oder stationär) behandelt. Um einen größeren Teil der Alkoholabhängigen durch suchtspezifische Angebote zu erreichen, wurden im Bereich der Alkoholabhängigkeit vor allem Konzepte zur Sekundärprävention in Einrichtungen der medizinischen und psychosozialen Basisversorgung entwickelt [2-4]. Während für Konsumenten illegaler Drogen in einem beträchtlichen Ausmaß auch niedrigschwellige Behandlungsund Hilfsangebote erprobt und ausgebaut wurden $[5,6]$, besteht im Bereich der niedrigschwelligen Behandlungsangebote für Alkoholabhängige noch ein erheblicher Nachholbedarf.

Nachdem eine Vielzahl positiver Erfahrungsberichte zum Einsatz der Akupunktur in der Behandlung von Suchterkrankungen veröffentlicht wurden und die Akupunktur ein fester Bestandteil US-amerikanischer Drogengerichte wurde [7], gibt es auch in Deutschland zunehmend Bestrebungen, diese Behandlungsmethode für Patienten mit Abhängigkeitserkrankungen einzusetzen. Die Vorteile dieser niedrigschwelligen Behandlungsmethode liegen darin, dass sie ohne Medikamente auskommt und als nonverbales Verfahren anfangs auch nur eine geringe Bereitschaft zur kognitiven Auseinandersetzung mit dem Alkoholproblem erfordert. Sie stellt erheblich weniger Anforderungen an den Klienten als andere Behandlungsformen und könnte somit Alkoholabhängigen den Schritt zur Inanspruchnahme weiterer suchtspezifischer 
Hilfen erheblich erleichtern. Die Suchtakupunktur wäre zudem eine kostengünstige Behandlungsalternative.

Die ersten Berichte über den Einsatz der Akupunktur in der Suchtbehandlung wurden von Wen und Cheung 1973 veröffentlicht [8,9]. Schon zu dieser Zeit wurden auch in Deutschland die ersten klinischen Erfahrungen mit diesem Verfahren gesammelt (vgl. [10]). Inzwischen liegen viele Berichte über den erfolgreichen Einsatz der Akupunktur in der stationären und ambulanten Behandlung verschiedener stoffgebundener und auch in der Behandlung nicht stoffgebundener Abhängigkeiten (Essstörungen, Spielsucht etc.) vor (vgl. [10-12]). Trotz der positiven klinischen Erfahrung fehlt jedoch bis heute der wissenschaftlich eindeutige Nachweis der berichteten Behandlungseffekte.

Für den Bereich der akupunkturgestützten Behandlung von Alkoholabhängigen werden von der Mehrzahl der Studien positive Ergebnisse im Hinblick auf die Outcome-Kriterien körperliches und psychisches Befinden, Rückfallvermeidung, Haltequote und Trinkgewohnheiten berichtet. In zwei dieser Arbeiten wurde die Suchtakupunktur als alleinige Behandlungsmethode eingesetzt $[13,14]$, in allen anderen Arbeiten zusammen mit medikamentösen [15-17] und psychotherapeutischen Behandlungsmethoden [18] oder als ambulante Weiterbehandlungsmaßnahme nach Entzug mit herkömmlichen Behandlungsmethoden [19,20].

In vier Studien wurden Kontrollgruppen mit einer Plazebobedingung (Scheinakupunktur) in die Untersuchung einbezogen. Die Ergebnisse dieser Studien zeigen nur zum Teil eine Überlegenheit der Akupunktur gegenüber der Plazebobedingung. In den Studien von Bullock und Mitarbeitern [19,20], die den Einsatz der Suchtakupunktur als Weiterbehandlungsmaßnahme nach herkömmlicher Entzugsbehandlung untersuchten, wurden nach 1, 3 und 6 Monaten signifikant bessere Ergebnisse in der echten Akupunkturgruppe hinsichtlich des Verlangens nach Alkohol, der Anzahl der Trinkepisoden, der Häufigkeit der Einweisungen in stationäre Entzugsbehandlungseinrichtungen und der Haltequote gefunden. In einer jüngeren Studie von Worner [21] mit ähnlichem Design waren die mit der Akupunkturbehandlung erzielten Ergebnisse der Scheinbehandlung jedoch nicht überlegen. Obwohl diese Studie aufgrund unterschiedlicher Bedingungen (Lokalisierung und Anzahl der gesetzten Akupunkturpunkte, Kontrollgruppenzusammensetzung, Outcome-Kriterien, Akupunktursetting u.a.) nur begrenzt mit den Studien von Bullock zu vergleichen ist, müssen die positiven Ergebnisse der älteren Studie nach diesen neueren Ergebnissen kritisch beurteilt werden.

Sapir-Weise et al. [16] untersuchten in einer weiteren plazebokontrollierten Studie den Einsatz der Ohrakupunktur als adjuvante Maßnahme zu einer herkömmlichen ambulanten Behandlung bestehend aus sozialer Unterstützung und/oder einer aversiven medikamentösen Behandlung mit Disulfiram oder Kalziumkarbimid. Auch in dieser Untersuchung wurde kein Unterschied zwischen der Experimental- und der Kontrollgruppe in den untersuchten Parametern Anzahl der Tage mit Alkoholkonsum und Craving gefunden.
Studien zum akupunkturgestützten Opiatentzug - bei den meisten handelt es sich um Untersuchungen mit Kontrollgruppendesign - zeigen als Trend eine mit medikamentösen Verfahren annähernd vergleichbare, aber nicht überlegene Wirkung des akupunkturgestützten Entzugs bezüglich der Outcome-Kriterien Konsumverhalten und Reduktion von Entzugssymptomen. Opiatabhängige Patienten werden in rein medikamentösen Behandlungsformen jedoch länger gehalten als in Behandlungsformen, die nur auf Akupunktur beruhen [22-25]. Mit der Akupunkturbehandlung von Kokain- und Crackabhängigen - auch in diesem Bereich handelt es sich bei den meisten um Untersuchungen mit Kontrollgruppendesign - können zwar positive Veränderungen im Hinblick auf die Konsumgewohnheiten, psychisches Befinden, Verlangen nach der Droge etc. erreicht werden, diese gehen jedoch ebenfalls nicht über die einer Plazebo-Akupunkturbehandlung hinaus [11,26-29].

Methodische Probleme, vor allem hohe Drop-out-Raten und fehlende oder ungeeignete Kontrollbedingungen, schwächen die Aussagekraft der Behandlungsergebnisse von Studien zur Suchtakupunktur. Eine kritische Bewertung der Ergebnisse wird auch durch eine 1990 [30] durchgeführte Metaanalyse von Studien zur Suchtakupunktur nahe gelegt, die zu dem Ergebnis kam, dass mit steigender methodischer Qualität eine Verschlechterung der beobachteten Behandlungsergebnisse einherging. Jedoch sind bei einer Verbesserung der methodischen Voraussetzungen der Studien nicht nur negative Effekte in Bezug auf die Behandlungsergebnisse zu erwarten. Während bei hohen Drop-out-Raten eher eine positive Selektion und damit eine Überschätzung der Behandlungsergebnisse $\mathrm{zu}$ erwarten ist, ist bei Kontrollbedingungen, die eine über Plazebo- und Suggestionseffekte hinausgehende Wirkung beim Patienten auslösen, eher eine Unterschätzung der spezifischen Effekte der Akupunktur zu erwarten. Da PlazeboAkupunkturverfahren - es handelt sich meistens um Einstechpunkte, die nur wenige Millimeter neben den „wahren“ Akupunkturpunkten liegen - eine mit dem Verum vergleichbare Wirkung hervorrufen können, kann zumindest nicht ausgeschlossen werden, dass die Wirksamkeit der Akupunktur in plazebokontrollierten Untersuchungen systematisch unterschätzt wird.

\section{Behandlung}

Seit 1998 wird in der Beratungsstelle Die Hummel des Sozialtherapeutischen Zentrums für Suchtkranke in Hamburg Hummelsbüttel eine ambulante Akupunkturbehandlung für Klienten mit einer Indikation zur Entzugsbehandlung und für Klienten in akuten Krisenzeiten (z. B. bei Rückfallgefahr oder nach einem Rückfall) angeboten. Mit der Suchtakupunktur steht den Klienten somit ergänzend zu den herkömmlichen Angeboten für Alkohol- und Medikamentenabhängige eine leicht erreichbare niedrigschwellige Behandlungsalternative in den Räumen der Beratungsstelle zur Verfügung.

Die Akupunktur wird in Gruppen von etwa 8-10 Klienten durchgeführt. Die Behandlung beginnt mit einer ärztlichen Eingangsuntersuchung und der Behandlungsplanung. Für die Entzugsbehandlung werden in den ersten zwei bis drei Wochen tägliche Sitzungen und anschließend über einen Zeitraum von zwei Monaten zwei bis drei Sitzungen pro Woche empfohlen. Der Konsum von Alkohol soll in den ersten 
Wochen schrittweise eingestellt werden. Für Klienten, die eine Krise mit der Gefahr eines Rückfalls oder nach einem Rückfall überwinden wollen, werden individuelle Verabredungen von bis zu 20 Sitzungen in drei Monaten getroffen. Der Abschluss von Behandlungsverträgen oder verbindliche Zusagen in Bezug auf die Einhaltung von Behandlungsvorgaben wurden von den Teilnehmern zum Zeitpunkt der Untersuchung nicht verlangt. Akupunkturklienten müssen jedoch ein Honorar von 10,- DM pro Sitzung bezahlen, das bei einer Kostenübernahme durch die Krankenkasse erstattet wird.

Die Behandlung wird nach dem im NADA-Protokoll [31] beschriebenen Verfahren mit einer Akupunktur von jeweils drei bis fünf Ohrpunkten je Ohr von Ärzten mit Suchtakupunkturausbildung durchgeführt. Während der 45 Minuten dauernden Akupunkturbehandlung sitzen die Klienten in bequemen Sesseln und hören entspannungsfördernde Musik. Die Ergebnisse der ärztlichen Untersuchungen, die wöchentliche Befragung der Klienten, wahrgenommene Termine und Besonderheiten werden kontinuierlich dokumentiert. Beratungsangebote und die Akupunkturbehandlung können in der Regel ohne Voranmeldung genutzt werden. Auch im weiteren Verlauf können die Angebote der Hummel dem Bedarf und den Bedürfnissen der Klienten entsprechend flexibel in Anspruch genommen werden.

\section{Fragestellung}

Mit der Akupunkturbehandlung sollen zum einen nachhaltige Behandlungseffekte hinsichtlich der Reduktion des Alkoholkonsums und der Verbesserung psychischer und somatischer Parameter erreicht werden, zum anderen sollen mit dieser Behandlungsform größere Teile derjenigen Alkoholabhängigen erreicht und im Suchthilfesystem gehalten werden, die durch andere Behandlungsmethoden nicht erreicht wurden. Die vorliegende Studie, die in der Zeit von Juni 1999 bis April 2000 von der Abteilung für Medizinische Psychologie des Universitätsklinikum Hamburg-Eppendorf im Auftrag der Martha-Stiftung durchgeführt wurde, hatte die Aufgabe, eine erste Einschätzung zur Umsetzbarkeit dieser Zielsetzung zu liefern und somit die Grundlage für die Konzeption einer umfassenderen Evaluationsstudie zu schaffen. In diesem Zusammenhang waren folgende Fragen zu beantworten:

1. Welche Merkmale kennzeichnen Akupunkturklienten im Unterschied zu Klienten anderer Beratungs- und Behandlungsangebote? Gibt es unterschiedliche Zugangswege und Selektionsprozesse? 2. In welchem Umfang können durch die Akupunkturbehandlung Veränderungen der Ergebnisvariablen Konsumverhalten, körperliches und seelisches Befinden erreicht werden? 3. Wie wird die Akupunktur in Anspruch genommen? Welchen Einfluss haben Behandlungsintensität und -dauer auf den Behandlungserfolg? 4. Welche Faktoren haben einen moderierenden Einfluss auf das Behandlungsergebnis? Unterscheiden sich Behandlungserfolge bei unterschiedlichen Therapiezielen und Diagnosen? Gibt es Klientengruppen, die unterschiedlich stark auf die Akupunkturbehandlung ansprechen (differenzielle Behandlungsindikation)? 5. Hat die Akupunkturbehandlung eine längerfristig anhaltende Wirkung und wie wirkt sich die Behandlung auf die Inanspruchnahme von Angeboten der Suchthilfe aus?
Ein Nachweis der spezifischen Wirkung der Akupunktur in Abgrenzung zu Plazeboeffekten und suggestiven Effekten, der nur mit kontrollierten klinischen Studien zu erbringen ist, wurde mit der vorliegenden Untersuchung nicht beabsichtigt.

\section{Methodik}

Die Datengrundlage der Studie bildete neben der seit Beginn der Akupunkturbehandlung von den Mitarbeitern der Hummel behandlungsbegleitend erhobenen Basisdokumentation eine Nachbefragung aller bis Ende 1999 behandelten Akupunkturklienten.

Folgende Klientenmerkmale wurden im Rahmen der Basisdokumentation $\mathrm{zu}$ Beginn der Behandlung mit einem Fragebogen bzw. im ärztlichen Interview erfasst: körperlicher Allgemeinzustand, psychische Störungen und Beeinträchtigungen, Konsumgewohnheiten, Krankengeschichte, Sozialsituation und Vorbehandlungen der Klienten. Der wöchentlich vorgelegte Fragebogen, der unverändert vom Akupunkturprojekt in der „Palette“ [13] übernommen wurde, enthielt Fragen zum aktuellen Befinden, zu Entzugserscheinungen, zum Konsumverhalten und zur Behandlungsmotivation. Der aktuelle körperliche Allgemeinzustand, das psychische Befinden, Depressivität und Angstzustände werden in diesem Fragebogen mit Hilfe von kurzen Selbsteinschätzungen der Klienten (z.B.: „Wie sehr haben Sie in der letzten Woche unter depressiven Verstimmungen gelitten?"; Antwortmöglichkeiten: „gar nicht“ 1 .... „stark“ 4) erfasst. Die Fragen zum Konsumverhalten orientieren sich am EuropASI [32]; die Fragen zur Entzugssymptomatik entstammen der Short Opiate Withdrawal Scale [33].

In der Zeit von Februar-April 2000 wurden alle bis zum Februar 2000 in der Hummel mit Akupunktur behandelten Klienten gebeten, einen per Post zugesandten Nachbefragungsbogen $\mathrm{zu}$ beantworten. Dieser Fragebogen enthielt Fragen zum aktuellen Konsumverhalten sowie zum psychischen und körperlichen Befinden, die in vergleichbarer Form auch behandlungsbegleitend erhoben wurden. Weiterhin wurden Fragen zu den Themenbereichen Behandlungsmotivation, Erleben der Behandlung und deren Wirkungen, Zufriedenheit mit der persönlichen und sozialen Situation, Inanspruchnahme von Angeboten der Suchthilfe gestellt.

\section{Ergebnisse der behandlungsbegleitend erhobenen Daten}

\section{Untersuchungsgruppe}

Zum Zeitpunkt der Studie lagen Daten über den Verlauf von 228 Klienten vor, die in der Hummel eine Akupunkturbehandlung in Anspruch genommen hatten. Der Frauenanteil der Stichprobe liegt über dem anderer ambulanter Einrichtungen des Hamburger Suchthilfesystems [34,35]. Dieser große Frauenanteil hängt einerseits vermutlich mit der Angebotspalette der Beratungsstelle, die spezielle Angebote für alkoholabhängige Frauen enthält, und mit einem großen Frauenanteil unter den therapeutisch tätigen Mitarbeitern der Hummel zusammen, ist andererseits möglicherweise aber auch auf die größere Akzeptanz der Behandlungsmethode bei Frauen zurückzuführen. 
Tab. 1 Stichprobenmerkmale im Vergleich

\begin{tabular}{llll}
\hline Merkmale & $\begin{array}{l}\text { Akupunktur- } \\
\text { klienten der } \\
\text { Hummel }\end{array}$ & $\begin{array}{l}\text { Beratungs- } \\
\text { klienten der } \\
\text { Hummel }\end{array}$ & BADO* \\
\hline $\begin{array}{l}\text { Geschlecht } \\
\text { weiblich } \\
\text { männlich }\end{array}$ & $42 \%$ & $35 \%$ & $30 \%{ }^{\prime}$ \\
$\begin{array}{l}\text { Durchschnittsalter } \\
\begin{array}{l}\text { Frauen } \\
\text { Männer }\end{array}\end{array}$ & $58 \%$ & $65 \%$ & $70 \%$ \\
$\begin{array}{l}\text { Bezug von Sozialhilfe } \\
\text { Frauen }\end{array}$ & 42 & 46 & $44^{\prime}$ \\
$\begin{array}{l}\text { Männer } \\
\text { Alter bei Beginn des } \\
\text { regelmäßigen Konsums }\end{array}$ & $19,5 \%$ & 43 & $44^{\prime}$ \\
$\begin{array}{l}\text { Frauen } \\
\text { Männer }\end{array}$ & 30 & $12 \%$ & $15 \%$ \\
\hline
\end{tabular}

* Ambulante Suchthilfe in Hamburg. Statusbericht 1998I 1997II zur Hamburger Basisdatendokumentation im ambulanten Suchthilfesystem

Akupunkturklientinnen und -klienten der Hummel bilden zwei Gruppen, die sich von Klientinnen und Klienten aus vergleichbaren ambulanten Beratungs- und Behandlungseinrichtungen in den Merkmalen Lebensalter, Sozialstatus und Einbindung in familiäre Zusammenhänge jeweils in genau entgegengesetzter Richtung unterscheiden: Klientinnen sind älter, Klienten jünger; Klientinnen haben einen besseren, Klienten einen schlechteren Sozialstatus; Klientinnen sind weniger, Klienten stärker in familiäre Zusammenhänge eingebunden. Diese geschlechtsspezifischen Differenzen sprechen grundsätzlich für unterschiedliche Zugangswege und Selektionsprozesse von Akupunkturklientinnen und -klienten.

Der für die Gesamtgruppe der Akupunkturklienten im Vergleich zu Klienten anderer ambulanter Hilfsangebote höhere Sozialstatus, der große Anteil allein stehender Frauen und die bei Frauen geringere Kinderzahl entsprechen weitgehend dem Charakter des Einzugsgebiets der Hummel und sind vermutlich nicht durch Selektionseffekte der Akupunkturbehandlung bedingt.

Nach den Ergebnissen des ärztlichen Interviews traten schwere depressive Zustände bei $31 \%$ der Klienten und schwere Angst- und Spannungszustände bei 36\% der Klienten im Jahr vor Behandlungsbeginn auf. Das Vorkommen psychischer Beeinträchtigungen liegt damit etwa im Mittelbereich der Prävalenzraten (22-68\%) für Symptome von
Angststörungen bei Alkoholabhängigen [36]. Da die Lebensprävalenz von Suizidversuchen bei Akupunkturklienten der Hummel mit 40\% für Frauen und 30\% für Männer weit höher als die für Alkoholabhängige geschätzte Prävalenz (vgl. [37]) liegt, muss davon ausgegangen werden, dass nach den vorliegenden Angaben bei einem großen Teil der Akupunkturklienten ernst zu nehmende psychische Störungen vorliegen und ein starker Bedarf an psychotherapeutischer Unterstützung besteht.

Für den weitaus größten Teil der Akupunkturklienten der Hummel (83,3\%) ist Alkohol das Hauptsuchtmittel. Alle weiteren Suchtmittel sind mit weniger als 5\% in der Stichprobe vertreten. Knapp 25\% konsumieren andere Drogen neben dem Hauptsuchtmittel. Dabei handelt es sich hauptsächlich um Medikamente, Haschisch und Kokain, aber auch um Heroin $(4 \times)$. Im Durchschnitt konsumierten die Akupunkturklienten an 18 von 30 Tagen vor Behandlungsbeginn Alkohol in irgendeiner Form. Bis zur Trunkenheit wurde von den Klienten im Durchschnitt an 11 von 30 Tagen getrunken, also an jedem dritten Tag.

Die mit der Akupunkturbehandlung beabsichtigte Zielsetzung war für $43 \%$ der Klienten nicht eindeutig zuzuordnen, da von den Akupunkturärzt(inn)en Verlaufsziele genannt werden z.B. erst Entgiftung, dann Stabilisierung. Eine Entgiftung hatten 36\% der Behandlungen zum Ziel, 15\% wollten einem Rückfall vorbeugen und $6 \%$ suchten Hilfe nach einem vor kurzem erlebten Rückfall.

\section{Inanspruchnahme}

Die empfohlene Standardbehandlung für den Entzug sieht tägliche Sitzungen über zwei bis drei Wochen und anschließend bis zu zwei Monate andauernd zwei bis drei Sitzungen pro Woche bei langsamem Ausschleichen des Alkoholkonsums vor. Dies entspricht einer optimalen Behandlungsdauer von etwa 25 Sitzungen (bei Stabilisierung werden individuelle Verabredungen getroffen: bis zu 20 Sitzungen in drei Monaten). Dieser empfohlene Behandlungsstandard wird jedoch nur von einer Minderheit der Klienten erreicht: 28\% der Klienten haben nur bis zu drei Sitzungen in Anspruch genommen, $51 \%$ bis zu 8 Sitzungen (MW 11,0; Range 1-41) und die Behandlungsdauer beträgt für etwa $30 \%$ der Klienten weniger als eine Woche und für $55 \%$ weniger als vier Wochen (MW 4,8; Range 0-30). Die Haltequote nimmt dementsprechend im Laufe der Behandlung stark ab, so dass in der 3. Behandlungswoche nur noch etwa $60 \%$ und in der 7 . Woche ein Drittel in der Behandlung verbleiben.

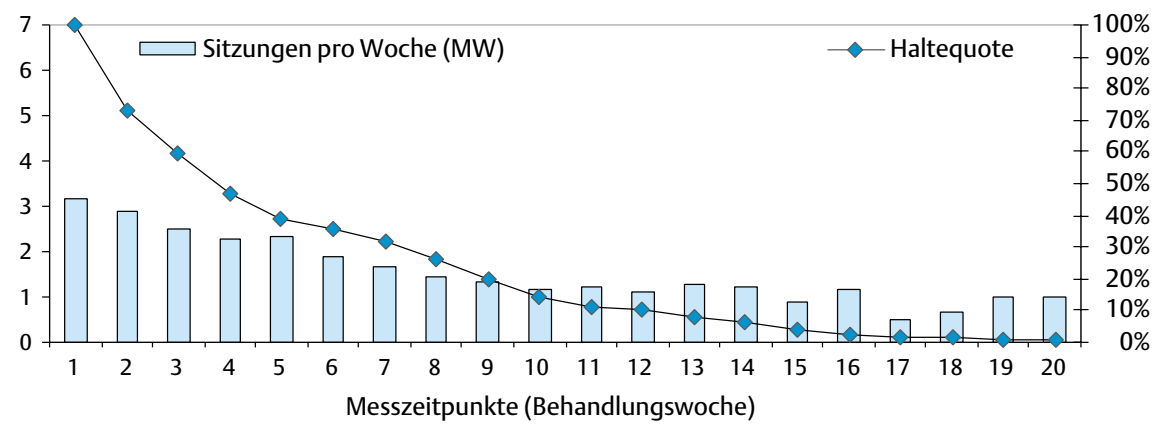

Abb. 1 Haltequote und Behandlungsfrequenz im Therapieverlauf. 


\section{Behandlungsergebnisse im Verlauf}

Veränderungen der zur Messung des Behandlungserfolgs in der Basisdokumentation wöchentlich erhobenen Variablen körperlicher und psychischer Allgemeinzustand, depressive Verstimmungen, Angstzustände, Entzugssymptome und Konsumverhalten wurden aufgrund der interindividuell stark streuenden Behandlungsdauer nach zwei prinzipiell unterschiedlichen Verfahren ausgewertet. Zum einen wurden die an zwei (bzw. drei) ausgewählten Messzeitpunkten dokumentierten Daten verglichen - gewählt wurden die Zeitpunkte: Behandlungsbeginn $\left(t_{0}\right)$ und 3 . Behandlungswoche $\left(t_{1}\right)$ sowie Behandlungsbeginn und 3. $\left(t_{1}\right)$ und 8 . Behandlungswoche $\left(t_{2}\right)$ - zum anderen wurde ein Vergleich von Behandlungsausgangswerten und -endwerten vorgenommen. Die erste Variante hat den Vorteil eines Vergleichs von individuellen Messwerten mit identischen Zeitintervallen. Der wesentliche Nachteil dieser Variante liegt darin, dass abhängig von der Wahl der Messzeitpunkte und den damit zusammenhängenden Behandlungsabbrüchen (bzw. -beendigungen) nur Teilstichproben aller behandelten Klienten analysiert werden können. Die zweite Variante bietet den Vorteil, dass alle Behandlungsverläufe, für die Daten von mindestens zwei Messzeitpunkten vorliegen $\left(\mathrm{t}_{0} /\right.$ Behandlungsende bzw. -abbruch), in die Analyse einbezogen werden können. Der Nachteil liegt in den interindividuell unterschiedlichen Zeitabständen zwischen den zu vergleichenden Messwerten.

Auf eine positive Veränderung aller erhobenen Ergebnisvariablen im Laufe der Behandlung weisen die statistisch signifikanten Messwertdifferenzen zwischen den Zeitpunkten $t_{0}$ und $t_{1}, t_{0}$ und $t_{1} / t_{2}$ und zwischen $t_{0}$ und Behandlungsende hin. Die stärksten Veränderungen treten in den ersten drei Behandlungswochen auf. Die positive Veränderung des Kon-
Tab. 2 Effektstärken der Veränderung Behandlungsbeginn/Ende

\begin{tabular}{|c|c|c|}
\hline \multirow[t]{2}{*}{ Ergebnisvariable } & \multicolumn{2}{|c|}{ Effektstärke $\left(\mathrm{ES}_{\text {prä}}\right)^{*}$ der Veränderung } \\
\hline & $\mathrm{t}_{0} /$ Bhdlgs.-ende & $t_{0} / t_{1}$ \\
\hline Angstzustände & 0,41 & 0,59 \\
\hline $\begin{array}{l}\text { Alkoholkonsum bis zur } \\
\text { Trunkenheit }\end{array}$ & 0,49 & 0,60 \\
\hline Entzugssymptome & 0,52 & 0,66 \\
\hline $\begin{array}{l}\text { körperlicher } \\
\text { Allgemeinzustand }\end{array}$ & 0,55 & 0,73 \\
\hline depressive Verstimmungen & 0,62 & 0,73 \\
\hline $\begin{array}{l}\text { Alkoholkonsum jeglicher } \\
\text { Gebrauch }\end{array}$ & 0,68 & 0,98 \\
\hline psychisches Befinden & 0,82 & 1,46 \\
\hline
\end{tabular}

* $\mathrm{ES}_{\text {prä }}=\left(M \mathrm{MW}_{\text {prä }}-\mathrm{MW} \mathrm{W}_{\text {post }}\right) / S D_{\text {prä }}$

sumverhaltens wird auch daran deutlich, dass von den Klienten, die bei Therapiebeginn noch trinken, am Ende der Akupunkturbehandlung etwas mehr als die Hälfte abstinent sind. Nur noch 6\% der Patienten geben am Ende der Therapie an, bis zur Trunkenheit zu konsumieren.

Mit zunehmender Behandlungsdauer summiert sich die Anzahl der Klienten, die die Behandlung beenden bzw. abbrechen. Für den Vergleich der Daten der Messzeitpunkte Behandlungsbeginn $\left(t_{0}\right)$, 3. Behandlungswoche $\left(t_{1}\right)$ und 8. Behandlungswoche $\left(t_{2}\right)$ stehen daher nur für $10 \%$ aller durchgeführten Behandlungen vollständige Daten zur Verfügung. Die in den ersten drei Behandlungswochen beobachtete positive Veränderung ist auch in den wenigen dokumentierten Behandlungsverläufen mit mindestens 8 Wochen Behandlungsdauer zu beobachten. Von der 3. bis
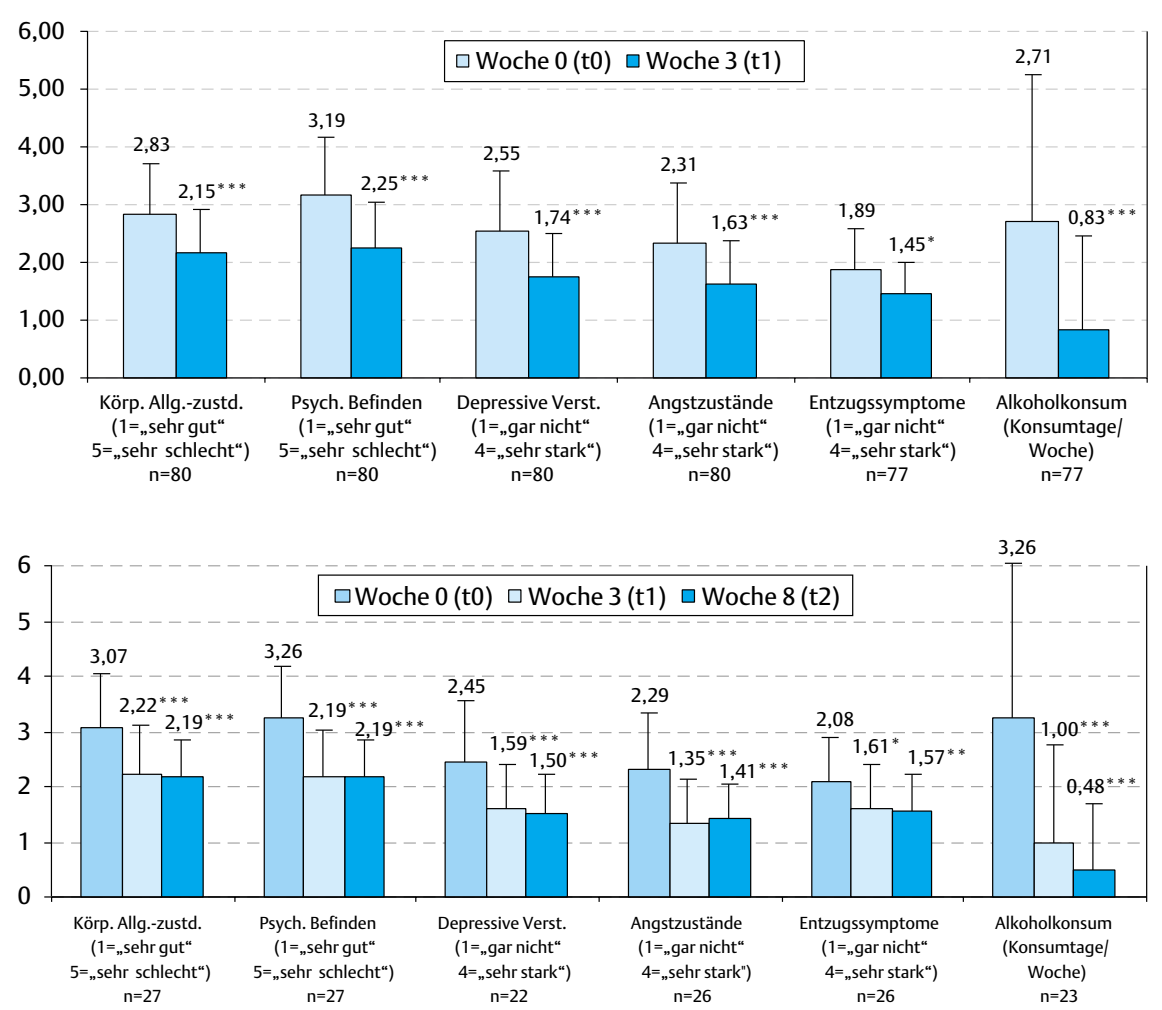

Abb. 2 Psychische und somatische Merkmale im Verlauf $\mathrm{t}_{0}$ und $\mathrm{t}_{1}$ (MW und SD).

Abb. 3 Psychische und somatische Merkmale im Verlauf $\mathrm{t}_{0}, \mathrm{t}_{1}$ und $\mathrm{t}_{2}$ (MW und SD). 
zur 8. Behandlungswoche treten kaum noch Veränderungen ein.

Die beobachteten Veränderungen liegen im Bereich mittlerer und großer Effektstärken und waren am stärksten bei Klienten mit den Therapiezielen Entgiftung und Stabilisierung nach Rückfall. Aufgrund der geringen bis sehr geringen Stichprobengröße von Klientengruppen mit unterschiedlichem Therapieziel sind die beobachteten Veränderungen nicht für alle Therapiezielgruppen signifikant.

Die positiven Veränderungen der Merkmale körperlicher und psychischer Allgemeinzustand, Depression, Angst und Entzugssymptome sind nicht unabhängig vom Therapieziel: Klienten mit den Therapiezielen Entgiftung, Stabilisierung nach Rückfall und mit „anderen“ (nicht einzuordnenden oder unbestimmten) Therapiezielen erleben eine stärkere Verbesserung ihrer Symptome als Klienten mit Therapieziel Rückfallprophylaxe.

Korrelationen zwischen den beobachteten Veränderungen der Ergebnisvariablen im Laufe der Therapie und der Behandlungsdauer sind nicht signifikant und erreichen Werte im Bereich von $r=-0,08$ bis $r=0,11$. Korrelationen zwischen den Veränderungswerten und der Häufigkeit der in Anspruch genommenen Akupunktursitzungen sind zwar statistisch signifikant, erreichen jedoch nur ein geringes Ausmaß (Werte zwischen $r=0,17$ bis 0,27 ).

Die Behandlungsdichte steht statistisch nachweisbar im $\mathrm{Zu}$ sammenhang mit folgenden Variablen: Alter $(r=0,27)$, Geschlecht (weiblich $>$ männlich), Therapieziel (Entgiftung $>$ Rückfallprophylaxe $>$ Stabilisierung nach Rückfall $>$ andere Therapieziele), Erwerbstätigkeit (in Ausbildung u.a. > in Rente $>$ erwerbstätig $>$ arbeitslos). Sie steht nicht im Zusammenhang mit den Variablen Alkoholkonsum und körperlicher Allgemeinzustand zu Beginn der Behandlung. Sie steht auch nicht im Zusammenhang mit der Entscheidung der Krankenkassen bezüglich der Kostenerstattung.

\section{Ergebnisse der Nachbefragung}

Die Behandlung lag zum Zeitpunkt der Nachbefragung 3,4-18,5 (MW = 10,5; SD = 4,2) Monate zurück. Von den 206 Klienten, denen der Fragebogen zugestellt werden konnte, haben $35,4 \%(n=73)$ den Fragebogen beantwortet zurückgeschickt. Die Rücklaufquote liegt somit etwas unter der anderer katamnestischer Untersuchungen. Die größeren Rücklaufquoten dieser Untersuchungen (37-70\%) wurden jedoch mit einem im Vergleich zu der hier vorgestellten Untersuchung erheblich höheren Erhebungsaufwand erzielt (Ankündigung der Katamnese, zusätzliche telefonische Nachbefragung etc., vgl. [38-41]).

\section{Untersuchungsgruppe}

Klienten, die an der Nachbefragung teilgenommen haben (Responder), und Klienten, die nicht teilnahmen (Non-Responder), unterscheiden sich mit Ausnahme der Frequenz der in Anspruch genommen Sitzungen in keinem der erhobenen Stichproben- und Behandlungsmerkmale signifikant.
Tab. 3 Stichprobenmerkmale von Respondern und Non-Respondern

\begin{tabular}{|c|c|c|}
\hline Merkmale & $\begin{array}{l}\text { Responder } \\
(n=73)\end{array}$ & $\begin{array}{l}\text { Non-Responder } \\
(n=132)\end{array}$ \\
\hline $\begin{array}{l}\text { Geschlecht } \\
\text { weiblich } \\
\text { männlich }\end{array}$ & $\begin{array}{l}51 \% \\
49 \%\end{array}$ & $\begin{array}{l}40 \% 1 \\
60 \%\end{array}$ \\
\hline $\begin{array}{l}\text { Durchschnittsalter } \\
\text { Frauen } \\
\text { Männer }\end{array}$ & $\begin{array}{l}47 \\
44\end{array}$ & $\begin{array}{l}46 \\
42\end{array}$ \\
\hline $\begin{array}{l}\text { soziale Situation* } \\
\text { Sozialhilfebezug } \\
\text { Ganztagsbeschäftigung } \\
\text { arbeitslos } \\
\text { Rente } \\
\text { Teilzeitbeschäftigung } \\
\text { Hausfrau/-mann }\end{array}$ & $\begin{array}{r}18,6 \% \\
39,5 \% \\
23,3 \% \\
11,6 \% \\
7,0 \% \\
14,0 \%\end{array}$ & $\begin{array}{r}9,3 \% \\
41,6 \% \\
33,8 \% \\
7,8 \% \\
5,2 \% \\
5,2 \%\end{array}$ \\
\hline $\begin{array}{l}\text { Behandlungsinanspruchnahme } \\
\text { Behandlungsdauer in Wochen } \\
\text { Behandlungstermine }\end{array}$ & $\begin{array}{r}6,4(0-29) \\
13,9(1-41)\end{array}$ & $\begin{array}{l}4,9(0-31) \\
9,7^{* *}(0-33)\end{array}$ \\
\hline
\end{tabular}

Ein besserer körperlicher Allgemeinzustand, die geringere Anzahl alkoholbedingter Folgeerkrankungen und ein größerer Anteil trocken beginnender Klienten - Differenzen unterhalb der statistischen Signifikanzgrenze - deuten dennoch zusammengenommen auf eine etwas günstigere körperliche und suchtbezogene Ausgangssituation bei Respondern hin.

\section{Beendigung der Behandlung}

Die Mehrheit der Klienten (61\%), die an der Nachbefragung teilnahmen, beenden die Behandlung, bevor sie die als Behandlungsstandard vorgesehenen 20 Akupunktursitzungen wahrgenommen haben. Ein Drittel der Klienten, die die Behandlung vorzeitig beenden, nennt als Anlass für die Beendigung persönliche Gründe, für jeweils ein Fünftel war zum Zeitpunkt der Beendigung der Therapieerfolg schon erreicht oder kein Erfolg erkennbar, 16\% nannten keine Gründe. Für ein Fünftel der Klienten mit vorzeitiger Beendigung der Therapie war nach eigener Einschätzung das Therapieziel schon bei einer Therapiedauer, die erheblich unter der vorgesehenen Standardbehandlung lag (MW 6,6 Sitzungen), erreicht. Nur für einen kleinen Teil (20-30\%) der

Tab. 4 Vorzeitige Beendigung der Akupunkturbehandlung $(n=73)$

\begin{tabular}{lr}
\hline Gründe & Anteil \\
\hline & \\
persönliche Gründe & $30 \%$ \\
Therapieziel schon erreicht & $19 \%$ \\
kein Erfolg erkennbar & $19 \%$ \\
Behandlung woanders fortgesetzt & $7 \%$ \\
keine Kostenübernahme & $5 \%$ \\
Akupunktur nicht geeignet & $5 \%$ \\
keine Angabe & $16 \%$ \\
\hline \hline
\end{tabular}




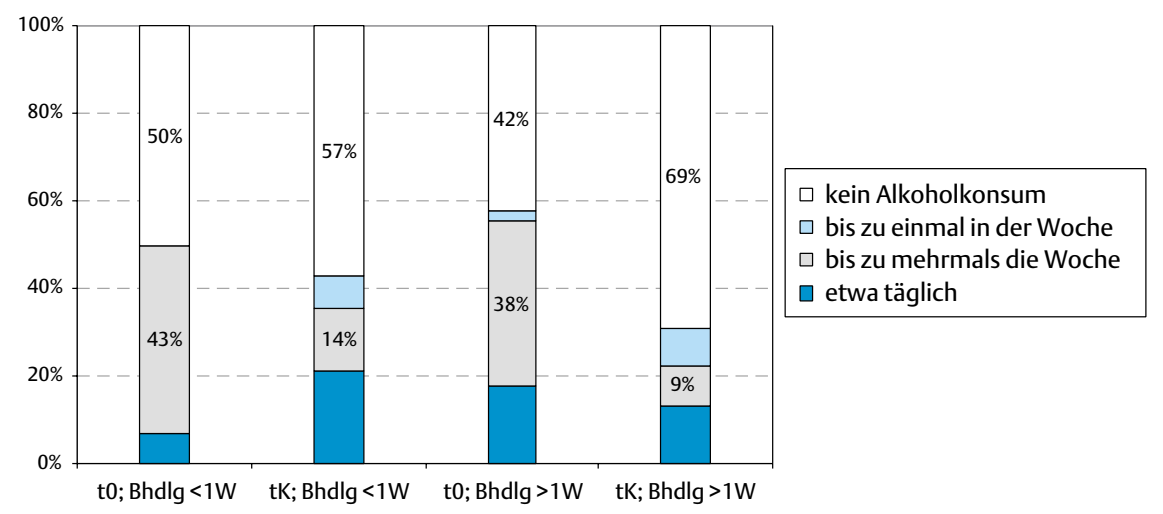

Abb. 4 Alkoholkonsum (jeglicher Gebrauch) im Monat vor Behandlungsbeginn $\left(t_{0}\right)$ und im Monat vor dem Katamnesezeitpunkt $\left(t_{K}\right)$.
Klienten liegt der Grund für den Behandlungsabbruch in negativen Erfahrungen mit der Akupunktur.

\section{Konsumverhalten im Monat der Nachbefragung}

Der erreichte Status des Konsumverhaltens am Ende der Behandlung konnte im Katamnesezeitraum nicht in vollem Ausmaß gehalten werden. Dennoch trinken die befragten Klienten zum Katamnesezeitpunkt weniger als zu Beginn der Behandlung: Über die Hälfte der Klienten mit Therapieziel Entzug/Entwöhnung (57\%) und ebenfalls über die Hälfte der Klienten mit nicht eindeutig zugeordnetem Therapieziel (60\%) - es handelt sich hierbei um Klienten, die zu Beginn der Behandlung regelmäßig Alkohol getrunken hatten - leben zum Katamnesezeitpunkt abstinent; nur noch 15 bzw. 17\% dieser Klienten konsumieren täglich Alkohol. Von den Klienten mit Therapieziel Rückfallprophylaxe wurde bis zum Katamnesezeitpunkt ein Viertel rückfällig.

Eine multivariate Varianzanalyse des Messwiederholungseffektes $\left(t_{0}=\right.$ Behandlungsbeginn; $t_{K}=$ Zeitpunkt der Katamnese) mit den Faktoren Behandlungsziel - geprüft wurden die Gruppen mit Therapieziel Entzug/Entwöhnung und „andere Ziele" - und Leistungsinanspruchnahme mit den Stufen: NonStarter ( $<1,0$ Behandlungswochen), Beender ( $>20$ Sitzungen) und Starter (alle Übrigen) ergab einen signifikanten Zeiteffekt sowie einen signifikanten Wechselwirkungseffekt zwischen Zeit und Gruppe der Leistungsinanspruchnahme sehr hoher Effektstärke (Entzugsklienten: Zeit: $\mathrm{p}<0,001, \eta^{2}=0,587$; Gruppe $\times$ Zeit: $p=0,004, \eta^{2}=0,259$ bzw. „Andere“: Zeit: $p=0,001$, $\eta^{2}=0,674$; Gruppe $\times$ Zeit: $\left.p=0,010, \eta^{2}=0,464\right)$. Verbesserungen im Trinkverhalten sind demnach auch zum Katamnesezeitpunkt noch signifikant. Dabei ist die Reduktion des Alkoholkonsums in der Klientengruppe mit Therapieziel Entzug/Entwöhnung bei „Beendern“ stärker als bei „Startern“ und bei diesem wiederum stärker als bei „Non-Startern“. Diese Effekte bleiben auch unter Berücksichtigung der Katamnesedauer als Kovariate stabil.

\section{Körperlicher Allgemeinzustand und psychisches Befinden}

Der am Ende der Behandlung erreichte Status des körperlichen Allgemeinzustandes und des psychischen Befindens verschlechtert sich im Katamnesezeitraum für die Gesamtgruppe der Akupunkturklienten im Unterschied zum Konsumstatus nicht. Auch gibt es für diese Variablen im Unterschied zum Konsumstatus keine differenziellen Effekte für Klienten mit unterschiedlichen Therapiezielen. Eine Prüfung der Effekte der Akupunkturbehandlung mit Hilfe multivariater Varianzanalysen bestätigt den Effekt des Zeitfaktors $\left(t_{0} / t_{K}\right)$ und den Effekt des Faktors Leistungsinanspruchnahme (körperlicher Allgemeinzustand - Zeit: $p=0,002, \eta^{2}=0,146$ Gruppe: $\quad p=0,006, \quad \eta^{2}=0,146$; psychisches Befinden - Zeit: $\mathrm{p}<0,000, \eta^{2}=0,231$ Gruppe: $\left.\mathrm{p}=0,002, \eta^{2}=0,177\right)$. Beender hatten in beiden Variablen die besten Ausgangswerte und verbesserten sich über die Zeit am stärksten. Non-Starter hatten die schlechtesten Ausgangswerte, verbesserten sich jedoch ebenso wie Starter. Die Wechselwirkungseffekte waren jedoch statistisch nicht signifikant. Auch für die Variablen körperlicher Allgemeinzustand und psychisches Befinden blieben die Ergebnisse unter Berücksichtigung der Katamnesedauer als Kovariate stabil.

Tab. 5 Inanspruchnahme von Behandlungs- und Hilfsangeboten der Akupunkturteilnehmer im Katamnesezeitraum

\begin{tabular}{lc} 
Maßnahme & Anteil \\
\hline Beratung/Selbsthilfe & $44 \%$ \\
Entgiftung & $20 \%$ \\
keine Behandlung in Anspruch genommen oder geplant & $16 \%$ \\
Entwöhnung & $10 \%$ \\
Behandlung geplant & $10 \%$ \\
\hline \hline
\end{tabular}

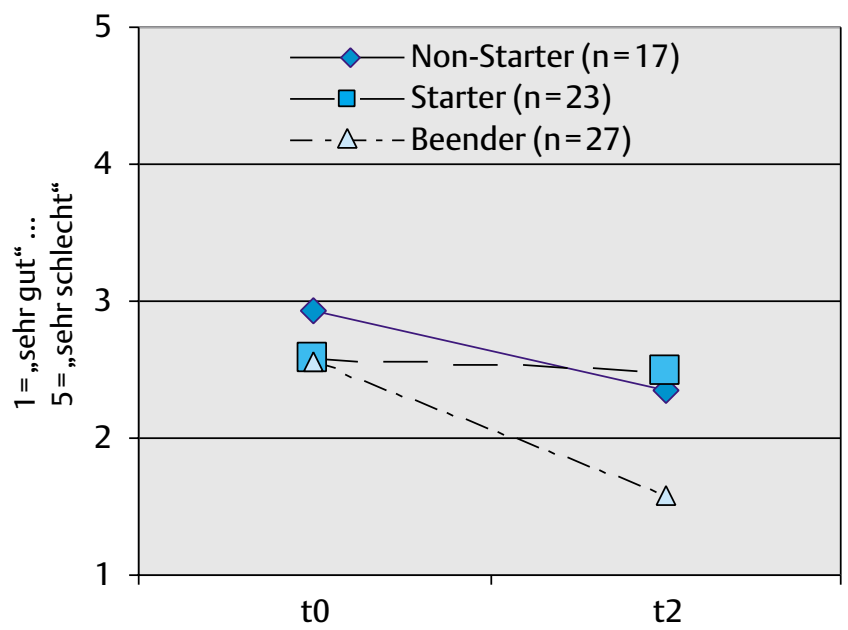

Abb. 5 Körperlicher Allgemeinzustand bei Beginn der Behandlung $\left(\mathrm{t}_{0}\right)$ und im Monat vor der Nachbefragung $\left(\mathrm{t}_{2}\right)$. 


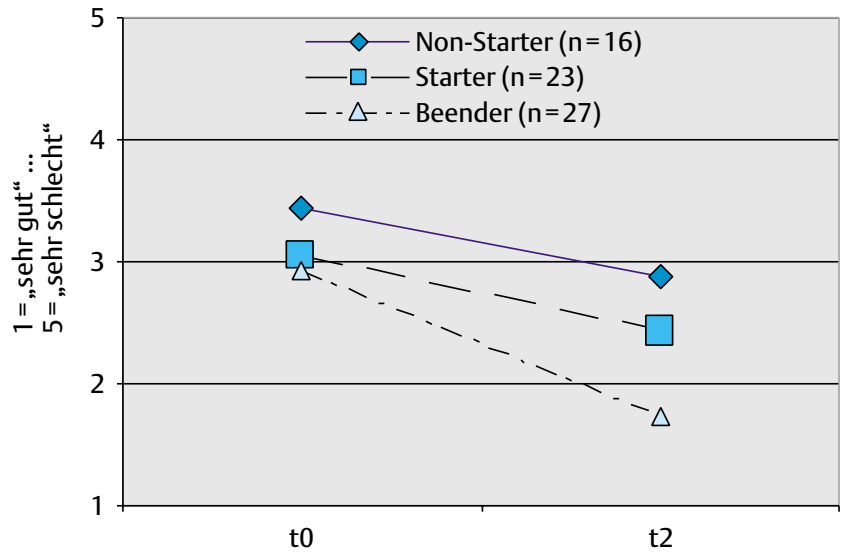

Abb. 6 Psychisches Befinden bei Beginn der Behandlung $\left(\mathrm{t}_{0}\right)$ und im Monat vor der Nachbefragung $\left(t_{2}\right)$.

Die Akupunkturklienten wurden katamnestisch befragt, welche von 9 vorgegebenen, häufig berichteten Wirkungen der Akupunktur sie nach der Behandlung an sich beobachten konnten. Am häufigsten wurde eine größere innere Ausgeglichenheit (68\%), eine größere Zuversicht in Bezug auf die Bewältigung der Suchtproblematik (55\%) und eine geringere Neigung zur Stressreaktion (44\%) erlebt. Die anderen vorgegebenen Wirkungen - „ehrlicherer Umgang mit sich selbst“, „stärkere Beachtung eigener Empfindungen/eigener Wünsche“, „größere Offenheit gegenüber anderen/gegenüber weiteren Hilfsangeboten“ und eine Verminderung von Stimmungsschwankungen - wurden jeweils von etwa einem Drittel angekreuzt. Die freien Antworten zur Wirkung der Akupunktur deuten wie die vorgegebenen Antworten auf eine entspannende, ausgleichende und psychisch stabilisierende Wirkung der Akupunktur als zentralen subjektiv empfundenen Effekt hin.

Die Akupunktur scheint nach den Ergebnissen der Nachbefragung auch einen positiven Einfluss auf die Inanspruchnahme weiterführender Hilfen zu haben: Nur 16\% der befragten Klienten hatten bis zum Zeitpunkt der Nachbefragung keine weiterführende suchtspezifische Behandlung oder Hilfe in Anspruch genommen bzw. geplant.

\section{Diskussion}

Die vorliegende Untersuchung hatte zu prüfen, inwieweit die an die Akupunkturbehandlung als niedrigschwelliges Behandlungsangebot gestellten Erwartungen erfüllt werden können. Die Erwartungen richten sich zum einen auf eine Reduktion des Alkoholkonsums, zum anderen aber auch auf die Wirkung der Akupunktur auf das Inanspruchnahmeverhalten von Alkoholabhängigen, die bisher nicht oder nicht ausreichend von den Angeboten des Suchthilfesystems erreicht wurden, sowie auf die Funktion der Akupunktur zur längerfristigen Integration der Klienten in das Suchthilfesystem. Die Datenbasis für die Untersuchung bildete neben einer von Beginn des Akupunkturprojekts an behandlungsbegleitend geführten Dokumentation basaler Daten des Behandlungsverlaufs auch eine postalische Nachbefragung aller bis Dezember 1999 in der Beratungs- und Behandlungsstelle Die Hummel behandelten Akupunkturklienten.
Akupunkturklienten der Hummel unterscheiden sich von Klienten anderer Beratungs- und Behandlungseinrichtungen durch einen besonders hohen Frauenanteil. Die große Akzeptanz des Angebots bei weiblichen Alkoholabhängigen ist vermutlich auf die besondere Berücksichtigung von frauenspezifischen Angeboten in der Hummel zurückzuführen, sie lässt aber auch vermuten, dass die Akupunkturbehandlung als „alternative Behandlungsform“ generell eine stärkere Resonanz bei Frauen findet. Trotz der unterschiedlichen Attraktivität des Akupunkturangebots bei Frauen und Männern hat das Geschlecht nach den Ergebnissen der Untersuchung keinen Einfluss auf den Erfolg der Behandlung. Auch andere Patientenmerkmale, die sich bei Frauen und Männern wahrscheinlich aufgrund geschlechtsspezifischer Zugangswege unterscheiden, standen nicht im Zusammenhang mit dem Behandlungserfolg.

Auch wenn Vergleichsdaten fehlen, die eine Beurteilung der Schwere der Abhängigkeitsproblematik im Vergleich zu anderen Behandlungsformen ermöglichen, so ist aufgrund des niedrigschwelligen Charakters des Behandlungsangebots doch $\mathrm{zu}$ vermuten, dass eher Alkoholabhängige mit einem vergleichsweise geringen Alkoholkonsum durch die Akupunkturbehandlung erreicht werden. Der große Anteil von psychischen Beeinträchtigungen bis hin zu häufig berichteten Suizidgedanken und -versuchen unter den Akupunkturklienten und die bei Akupunkturklienten verglichen mit Beratungsklienten der Hummel größere Dauer der Alkoholabhängigkeit deuten jedoch auf eine Klientengruppe mit starker Abhängigkeitsproblematik und einem dringenden Behandlungs- und Unterstützungsbedarf hin.

Die Behandlungsdauer und die Häufigkeit der in Anspruch genommenen Akupunktursitzungen ist interindividuell sehr unterschiedlich. Gemeinsames Merkmal des Inanspruchnahmeverhaltens ist eine Haltequote, die in den ersten drei bis vier Behandlungswochen, in denen auch die wichtigsten Veränderungen stattfinden, am stärksten abfällt. Dieser stark abfallende Verlauf der Haltequote wurde auch in anderen Studien beobachtet und scheint charakteristisch für niedrigschwellige Behandlungsangebote zu sein, die den Klienten ein weitgehend unverbindliches „Erproben“ der Behandlungsmethode ermöglichen.

Die im Behandlungsverlauf wöchentlich erhobenen Selbstratings der Klienten zum psychischen Befinden, zum körperlichen Allgemeinzustand, zu depressiven Verstimmungen, zu Angst- und Spannungszuständen, zum Erleben von Entzugssymptomen und zum Alkoholkonsum lassen eine statistisch signifikante positive Veränderung dieser subjektiven Maße erkennen. Die Behandlungsdauer hatte nach den Ergebnissen der behandlungsbegleitend erhobenen Daten allein keinen nachweisbaren Einfluss auf den Behandlungserfolg. Der Erfolg wurde, wenn auch in geringem Maße, von der Frequenz der in Anspruch genommenen Akupunktursitzungen beeinflusst. Die Ergebnisse zeigen auch, dass schon mit einer vergleichsweise geringen Behandlungsintensität zumindest kurzfristig positive Veränderungen erzielt wurden. In Ergänzung dazu deuten die katamnestischen Ergebnisse zum Zusammenhang von Behandlungserfolg und -intensität darauf hin, dass längerfristig eine höhere Behandlungsintensität bei einem Teil der Klienten mit einem nachweisbar besseren Behandlungserfolg einhergeht, dass andererseits jedoch eine andere Teil- 
gruppe der Klienten schon bei einer kurzen Behandlungsdauer subjektiv einen Behandlungserfolg erlebt hat.

Aufgrund der Studienergebnisse wurde inzwischen ein finanzielles Anreizsystem zur Förderung der Haltequote in den ersten drei Behandlungswochen in der Hummel eingeführt. Da nach den bisherigen Untersuchungsergebnissen für einen Teil der Klienten auch bei einer Unterschreitung der vorgesehenen Behandlungsdauer Erfolge eintreten, sollte darüber hinaus in Zukunft nach Möglichkeiten einer stärker an individuellen Bedingungen und Zielen orientierten Behandlungsplanung gesucht werden, die dann hinsichtlich ihrer Wirkung auf den Behandlungserfolg zu prüfen wären.

Während positive Veränderungen von körperlichen und seelischen Befindlichkeitsmaßen in den Selbstaussagen der Patienten ablesbar waren, kann die Bedeutung der Akupunkturbehandlung als Behandlungsmodul im Rahmen des Suchthilfesystems und im Rahmen von Beratungseinrichtungen nur indirekt erschlossen werden. Auf eine positive, das Versorgungssystem für Alkoholabhängige ergänzende Wirkung der Akupunktur weisen folgende Ergebnisse der Studie hin:

- Für etwa ein Drittel der Klienten war die Akupunkturbehandlung die erste suchtspezifische Behandlung, die sie in ihrer Suchtkarriere in Anspruch genommen hatten.

- Mit Einführung der Akupunkturbehandlung hat die Anzahl von Klienten, die in der Hummel beraten wurden, um 36\% zugenommen.

- Nur 16\% der befragten Klienten hatten bis zum Zeitpunkt der Nachbefragung keine weiterführende suchtspezifische Behandlung oder Hilfe in Anspruch genommen bzw. geplant.

Da bei der geringen Rücklaufquote der Nachbefragung Selektionseffekte nicht ausgeschlossen werden können - „Responder“ hatten häufiger als „Non-Responder“ Behandlungstermine in Anspruch genommen und es ist nicht ausgeschlossen ist, dass längerfristig erfolgreichere Klienten in stärkerem Maße an der Nachbefragung teilgenommen haben - geben die Ergebnisse der Nachbefragung nur einen ersten Anhaltspunkt für längerfristig wirkende positive Effekte der Akupunkturbehandlung. Da auch die behandlungsbegleitende Erhebung noch methodische Schwächen aufweist - die Daten beruhen auf Selbstaussagen der Patienten und es fehlen verlässliche Daten von Vergleichs- oder Kontrollgruppen sind weitere behandlungsbegleitende und katamnestische Studien zur methodischen Absicherung der ermutigenden ersten Ergebnisse zu kurz- und längerfristigen Effekten der ambulanten Suchtakupunktur unverzichtbar.

Die Effektivität herkömmlicher Behandlungsformen des Alkoholismus ist unbestritten. Das wesentliche Problem der Versorgung Alkoholabhängiger ist jedoch die geringe Inanspruchnahme der Angebote durch die Betroffenen. Daher sollte sich das Forschungsinteresse zukünftiger Untersuchungen stärker als bisher auf die Funktion der Suchtakupunktur im Rahmen des Versorgungssystems für Alkoholabhängige richten. Hierbei sollten insbesondere Fragen nach der Erreichbarkeit bisher nicht erreichter Alkoholabhängiger, nach der Akzeptanz der Akupunktur als Frühinterventionsform, nach differenziellen Indikationskriterien und nach der erzielten längerfristigen Bindung der behandelten Klienten in das
Suchthilfesystem systematisch nach Möglichkeit auch im Vergleich mit anderen (medikamentösen) ambulanten Behandlungsformen untersucht werden.

Längerfristig wird die Akupunktur den bis jetzt noch ausstehenden wissenschaftlichen Nachweis einer spezifischen, über Plazebo- und Suggestionseffekte hinausgehende Wirkung in aufwändigen kontrollierten klinischen Studien erbringen müssen, um als alternative Behandlungsform bestehen zu können. Unter den gegenwärtigen unbefriedigenden Versorgungsbedingungen für Alkoholabhängige könnte die Akupunkturbehandlung jedoch als ergänzende oder adjuvante niedrigschwellige Behandlungsmaßnahme im Zusammenwirken mit anderen Angeboten einen wichtigen Beitrag zur Verbesserung der Versorgungssituation für Alkoholabhängige und andere Suchtkranke leisten.

\section{Literatur}

${ }^{1}$ Feuerlein W, Küfner H, Soyka M. Alkoholismus - Mißbrauch und Abhängigkeit: Entstehung - Folgen - Therapie. Stuttgart: Thieme, 1998

2 John U, Hapke U, Rumpf H-J, Hill A, Dilling H. Prävalenz und Sekundärprävention von Alkoholmißbrauch und -abhängigkeit in der medizinischen Versorgung. Baden-Baden: Nomos Verlags-Gesellschaft, 1996

${ }^{3}$ John U, Veltrup C, Driessen M. Sekundärprävention der Alkoholabhängigkeit: Motivationsarbeit. Zeitschrift für Präventivmedizin und Gesundheitsförderung 1994; 6: 103-107

${ }^{4}$ Kremer G, Wienberg G, Dormann S, Pörksen N, Wessel T. Patienten mit Alkoholproblemen in der medizinischen Basisversorgung. Bedarfslage und Umsetzung eines Interventionskonzeptes in Arztpraxen und Allgemeinkrankenhäusern. In: Deutsche Hauptstelle gegen die Suchtgefahren (Hrsg). Alkohol, Konsum und Missbrauch. Alkoholismus, Therapie und Hilfe. Freiburg i. Br: Lambertus, 1997: 355-370

${ }^{5}$ Hoffmann-Bayer M. Die Herausforderung: Niedrigschwellige Konzepte in der ambulanten Drogenhilfe. In: Heckmann W (Hrsg). Drogentherapie in der Praxis. Ein Arbeitsbuch für die 90er Jahre. Suchtprobleme in Pädagogik und Therapie. Weinhem: Beltz, 1991: 240-259

${ }^{6}$ Schneider W. Niedrigschwellige Angebote und akzeptanzorientierte Drogenarbeit Vortragsmanuskript zum Internationalen Suchtkongress „Der Stellenwert der Suchtkrankheit im Gesundheitssystem“ 27.05.-01.06.1996 in Baden bei Wien. Wiener Zeitschrift für Suchtforschung, 1997; 20: 67-70

7 Tauber J. Das amerikanische Drogengericht. In: Baudis R (Hrsg). Punkte der Wandlung: Suchtakupunktur nach NADA-Protokoll. Rudersberg: Verlag für Psychologie, Sozialarbeit und Sucht, 1991: 237-249

${ }^{8}$ Wen $\mathrm{H}$, Cheung S. Treatment of drug addiction by acupuncture and electrical stimulation. Am J Med 1973; 9: 138-141

${ }^{9}$ Wen HL, Cheung SYC. Treatment of drug addiction by acupuncture and electrical stimulation. American Journal of Acupuncture 1973; 1: 71-75

10 Punkte der Wandlung: Suchtakupunktur nach NADA-Protokoll. In: Baudis R (Hrsg). Rudersberg: Verlag für Psychologie, Sozialarbeit und Sucht, 1999

${ }^{11}$ Lipton DS, Brewington V, Smith M. Acupuncture for crack-cocaine detoxification: experimental evaluation of efficacy. J Subst Abuse Treat 1994; 11: 205-215

12 Smith MO, Khan I. An acupuncture programme for the treatment of drug-addicted persons. Bull-Narc 1988; 40: 35-41 
${ }^{13}$ Verthein U, Raben R, v Soer J. Ambulante Akupunkturbehandlung bei Drogen- und Alkoholabhängigen - Ergebnisse einer Verlaufsuntersuchung. Sucht 2000; 46: 62-76

${ }^{14}$ Shentian S, Zhishun Y, Weibin G, Chunhui W, Furong D, Fengying G, Guozhi W, Yumei J, Yuying C. Clinical report of drinking intervention on 310 cases with auriculo-acupuncture. Journal of Traditional Chinese Medicine 1988; 8: 123-124

${ }^{15}$ Lewenberg A. Electroacupuncture and antidepressant treatment of alcoholism in a private practice. Clin-Ther 1985; 7: 611-617

${ }^{16}$ Sapir-Weise R, Berglund M, Frank A, Kristenson H. Acupuncture in alcoholism treatment: a randomized out-patient study. Alcohol and Alcoholism 1999; 34: 629-635

${ }^{17}$ Tuleweit U, Günsfelder C. Die medikamentenfreie Behandlung des Alkoholentzugssyndroms bei Alkoholkranken mit Akupunktur. Akupunktur, Theorie und Praxis 1998; 26: 83-85

${ }^{18}$ Gaa'l CL, Freebairn C. Ear-acupuncture relaxation therapy in alcoholics. Report on a follow-up survey. Med J Aust 1979; 2: 179-180

${ }^{19}$ Bullock ML, Umen AJ, Culliton PD, Olander RT. Acupuncture treatment of alcoholic recidivism: a pilot study. Alcohol-ClinExp-Res 1987; 11: 292-295

${ }^{20}$ Bullock ML, Culliton PD, Olander RT. Controlled trial of acupuncture for severe recidivist alcoholism. Lancet 1989; 1: 1435-1439

${ }^{21}$ Worner TM, Zeller B, Schwarz H, Zwas F, Lyon D. Acupuncture fails to improve treatment outcome in alcoholics. Drug-AlcoholDepend 1992; 30: 169-173

${ }^{22}$ Newmeyer JA, Johnson G, Klot S. Acupuncture as a detoxification modality. J Psychoactive Drugs 1984; 16: 241-261

${ }^{23}$ Washburn AM, Fullilove RE, Fullilove MT, Keenan PA, McGee B, Morris KA, Sorensen JL, Clark WW. Acupuncture heroin detoxification: a single-blind clinical trial. J Subst Abuse Treat 1993; 10 : 345-351

${ }^{24}$ Moner SE. Acupuncture and addiction treatment. J Addict Dis 1996; 15: 79-100

${ }^{25}$ Tennant, Jr FS. Outpatient heroin detoxification with acupuncture and staplepuncture. West J Med 1976; 125: 191-194

${ }^{26}$ Culliton PD, Kiresuk TJ. Overview of substance abuse acupuncture treatment research. J Altern Complement Med 1996; 2: 149-159 discussion 161-145

${ }^{27}$ Avants SK, Margolin A, Chang P, Kosten TR et al. Acupuncture for the treatment of cocaine addiction: Investigation of a needle puncture control. J Subst Abuse Treat 1995; 12: 195-205

${ }^{28}$ Otto KC, Quinn C, Sung YF. Auricular acupuncture as an adjunctive treatment for cocaine addiction. A pilot study. Am J Addict 1998; 7: 164-170

${ }^{29}$ Bullock ML, Kiresuk TJ, Pheley AM, Culliton PD, Lenz SK. Auricular acupuncture in the treatment of cocaine abuse. A study of efficacy and dosing. J Subst Abuse Treat 1999; 16: 31-38

${ }^{30}$ Ter Riet G, Kleijnen J, Knipschild P. A meta-analysis of studies into the effect of acupuncture on addiction. Br J Gen Pract 1990; 40: $379-382$

${ }^{31}$ Raben R. Einführung in die Ohrakupunktur nach dem NADAProtokoll. In: Baudis R (Hrsg). Punkte der Wandlung: Suchtakupunktur nach NADA-Protokoll. Rudersberg: Verlag für Psychologie, Sozialarbeit und Sucht, 1999: 16-37

${ }^{32}$ Kokkevi A, Hartgers C. EuropASI: European adaptation of a multidimensional assessment instrument for drug and alcohol dependance. European Addiction Research 1995; 1: 208-210

${ }^{33}$ Gossop M. The development of a short opiate withdrawal scale (SOWS). Addictive Behaviours 1990; 15: 487-490

${ }^{34}$ Schmid M, Vogt I, Simmedinger R. Ambulante Suchthilfe in Hamburg. Statusbericht 1997 zur Hamburger Basisdatendokumentation im ambulanten Suchthilfesystem. Frankfurt/M: ISSPONTIFEX, 1998
${ }^{35}$ Schmid M, Vogt I, Simmedinger R. Ambulante Suchthilfe in Hamburg. Statusbericht 1998 zur Hamburger Basisdatendokumentation im ambulanten Suchthilfesystem. Frankfurt/M: ISSPONTIFEX, 1999

${ }^{36}$ Kushner M, Sher K, Beitmann B. The relationship between alcohol problems and the anxiety disorders. Am J Psychiatry 1990; 147: 685-695

${ }^{37}$ Wünschmann B. Alkohol. Jahrbuch Sucht 92. Geesthacht: Neuland, 1991

${ }^{38}$ Brechner K, Shippee G, Obitz FW. Compliance techniques to increase mailed questionnaire return rates from alcoholics. J Stud Alcohol 1976; 37: 995-996

${ }^{39}$ Watzl H. Überlegungen zur Bewertung von Abstinenzraten. Suchtgefahren 1979; 1: 37-38

${ }^{40}$ Rink J. Probleme bei Untersuchungen zum Therapieerfolg im Suchtbereich. Suchtgefahren 1984; 30: 103-106

${ }^{41}$ Vannicelli M, Pfau B, Ryback RS. Data attrition in follow-up studies of alcoholics. J Stud Alcohol 1976; 37: 1325-1330

\section{Dr. rer. medic. Henry Buhk}

Universitätsklinikum Hamburg-Eppendorf

Medizinische Klinik und Poliklinik

Abteilung für Medizinische Psychologie

Pav. 69

Martinistraße 52

20246 Hamburg

E-mail: Hbuhk@uke.uni-hamburg.de 